\title{
ARTICLE
}

Received 19 May 2014 | Accepted 29 Oct 2014 | Published 11 Dec $2014 \quad$ DOI: 10.1038/ncomms6701

\section{Roquin binding to target mRNAs involves a winged helix-turn-helix motif}

\author{
Anja Schuetz ${ }^{1}$, Yasuhiro Murakawa², Eva Rosenbaum³ ${ }^{3}$ Markus Landthaler ${ }^{2}$ \& Udo Heinemann ${ }^{1,4}$
}

Roquin proteins mediate mRNA deadenylation by recognizing a conserved class of stem-loop RNA degradation motifs via their Roquin domain. Here we present the crystal structure of a Roquin domain, revealing a mostly helical protein fold bearing a winged helix-turn-helix motif. By combining structural, biochemical and mutation analyses, we gain insight into the mode of RNA binding. We show that the winged helix-turn-helix motif is involved in the binding of constitutive decay elements-containing stem-loop mRNAs. Moreover, we provide biochemical evidence that Roquin proteins are additionally able to bind to duplex RNA and have the potential to be functional in different oligomeric states.

\footnotetext{
${ }^{1}$ Helmholtz Protein Sample Production Facility, Max Delbrück Center for Molecular Medicine, 13092 Berlin, Germany. ${ }^{2}$ Laboratory for RNA Biology and Posttranscriptional Regulation, Berlin Institute for Medical Systems Biology, Max Delbrück Center for Molecular Medicine, 13092 Berlin, Germany. ${ }^{3}$ Structure and Membrane Interaction of G-Proteins, Max Delbrück Center for Molecular Medicine, 13092 Berlin, Germany. ${ }^{4}$ Chemistry and Biochemistry Institute, Freie Universität Berlin, 14195 Berlin, Germany. Correspondence and requests for materials should be addressed to U.H. (email: heinemann@mdc-berlin.de)
} 
B inding of proteins to distinct cis-regulatory elements within the untranslated region (UTR) of messenger RNAs (mRNAs) regulates the lifespan of these mRNAs and controls the gene expression at the post-transcriptional level. Roquin proteins, for example, were recently reported to specifically bind to a newly discovered conserved class of RNA stem-loop motifs, known as constitutive decay elements (CDE), which are present in the $3^{\prime}$ UTR of various mRNAs, thereby initiating their degradation. On a transcriptome-wide scale, $>50$ vertebrate CDEcontaining mRNAs were identified as Roquin targets, many of which encode proteins important for development as well as inflammation and immunity ${ }^{1}$. Roquin binding to the CDE in the $3^{\prime}$ UTR of tumour necrosis factor- $\alpha$ (TNF- $\alpha$ ) mRNA, for example, limits the synthesis of the proinflammatory cytokine TNF- $\alpha$ (ref. 1). The expression of ICOS (inducible T-cell costimulator), a gene encoding an essential receptor for follicular T-cells, is also restricted by Roquin, which promotes the degradation of ICOS mRNA, thereby playing an important role in autoimmunity ${ }^{2,3}$.

Mammalian genomes encode two roquin paralogs with seemingly redundant functions ${ }^{4,5}$. Human ROQUIN1, also known as $\mathrm{RC} 3 \mathrm{H} 1$ (ring finger and $\mathrm{CCCH}$-type zinc finger domains 1 ), is a 1,133 amino-acid protein, containing a RING (really interesting new gene)-type zinc finger, a roquin domain (ROQ), a CCCH-type zinc finger (zf), and a poorly defined carboxy (C)-terminal, proline-rich domain. Murine Roquin binding to CDE-like RNA stem-loop motifs was shown to be mediated by the ROQ domain, while the C-terminal effector domain recruits the Ccr4-Caf1-Not complex, resulting in Cafladependent deadenylation and consecutive degradation of CDEcontaining mRNAs ${ }^{1}$. Although Roquin proteins were shown to act broadly as mediators of mRNA deadenylation by recognizing a conserved class of stem-loop RNA degradation motifs via the ROQ domain ${ }^{1}$, the structural basis for stem-loop mRNA recognition is still unknown. To shed light on this, we determined the crystal structure of the ROQ domain of the human ROQUIN1 protein revealing a winged helix-turn-helix $(\mathrm{wHTH})$ motif. Additional biochemical and mutational analyses show that ROQUIN1 is able to bind both stem-loop and duplex RNA and that these interactions are mediated by the wHTH motif. Moreover, we provide evidences that ROQUIN1 has the potential to be functional in various oligomeric states and also provide a dimer model of the ROQ domain.

\section{Results}

Crystal structure of the ROQ domain. To obtain the crystal structure of the human ROQUIN1/RC3H1 protein, several constructs were used for crystallization. Although the produced proteins comprised up to three functional domains (RING, ROQ, zf domains), we always obtained the same degradation product, most likely representing the ROQ domain with all stably folded elements. We determined the crystal structure of this portion of ROQUIN1 at 1.91- $\AA$ resolution by single-wavelength anomalous diffraction phasing. The final model was refined to an $R_{\text {work }}$ of $20.0 \%$ and an $R_{\text {free }}$ of $24.1 \%$ (Table 1 , Supplementary Fig. 1a,b) and includes amino-acid residues $177-328$. The overall structure of the ROQ domain shows a compact $(\alpha+\beta)$ fold featuring seven $\alpha$-helices, one $310^{-}$-helix and a short three-stranded antiparallel $\beta$ sheet (Fig. 1a). The structure reveals a wHTH motif spanning from helix $\alpha 2$ to the $310^{\text {-helix }} \eta 1$, with helix $\alpha 1$ and helices $\alpha 5-\alpha 7$ from the ROQ domain packing against the wHTH motif (Fig. 1a,b, Supplementary Fig. 1a). The overall fold is stabilized by a salt bridge between the wHTH motif (R251 in helix $\alpha 4$ ) and a C-terminal residue of helix $\alpha 5$ (E293), and by hydrogen bonding interactions between the amino (N)-terminal helix $\alpha 1$ and helices $\alpha 2$ and $\alpha 4$ of the wHTH motif.

\begin{tabular}{|c|c|c|}
\hline & $\begin{array}{l}\mathrm{RC} 3 \mathrm{H} 1^{178-328} \\
\text { Pt derivative }\end{array}$ & RC3H1 177-328 native \\
\hline \multicolumn{3}{|l|}{ Data collection } \\
\hline Space group & $P 2_{1} 2_{1} 2$ & $\mathrm{C} 2$ \\
\hline \multicolumn{3}{|l|}{ Cell dimensions } \\
\hline$a, b, c(\AA)$ & $61.3,160.5,29.4$ & $169.7,29.8,60.2$ \\
\hline$\alpha, \beta, \gamma\left({ }^{\circ}\right)$ & $90,90,90$ & $90,100.9,90$ \\
\hline Resolution $(\AA)$ & $40.31-2.17(2.25-2.17)$ & $44.39-1.91(1.98-1.91)^{\star}$ \\
\hline$R_{\text {merge }}(\%)$ & $8.9(52.5)$ & $9.5(44.3)$ \\
\hline$<I / \sigma(I)>$ & $14.7(2.8)$ & $9.1(2.0)$ \\
\hline Completeness (\%) & $98.9(91.3)$ & $99.2(95.7)$ \\
\hline Redundancy & $7.3(4.4)$ & $3.2(2.9)$ \\
\hline \multicolumn{3}{|l|}{ Refinement } \\
\hline Resolution $(\AA)$ & 2.17 & 1.91 \\
\hline No. reflections & 117,110 & 75,166 \\
\hline$R_{\text {work }} / R_{\text {free }}(\%)$ & & $20.0 / 24.1$ \\
\hline No. atoms & & 2,564 \\
\hline Protein & & 2,403 \\
\hline Water & & 161 \\
\hline \multicolumn{3}{|l|}{ Average B-factor $\left(\AA^{2}\right)$} \\
\hline Overall & & 25.8 \\
\hline Protein & & 25.6 \\
\hline Water & & 29.0 \\
\hline \multicolumn{3}{|l|}{ R.m.s.d. } \\
\hline Bond lengths $(\AA)$ & & 0.016 \\
\hline Bond angles $\left({ }^{\circ}\right)$ & & 1.56 \\
\hline
\end{tabular}

R.m.s.d., root-mean-square deviation.

One single crystal was used to collect a complete dataset for each structure.

*Highest resolution shell is shown in parenthesis.

The canonical wHTH motif has originally been described to bind double-stranded DNA and consists of three $\alpha$-helices $(\mathrm{H} 1-\mathrm{H} 3)$, three $\beta$-strands (S1-3) and two characteristic loops ('wings') W1 and W2, arranged in the order H1-S1-H2-H3-S2W1-S3-W2 (ref. 6). Notably, in the crystal structure of the ROQ domain, the wHTH motif adopts a similar conformation with the exception that the W2-loop of the canonical wHTH motif is folded into the single-turn $3{ }_{10}$-helix $\eta 1$ (Fig. 1a,b). Moreover, the putative RNA-binding pocket is located within the wHTH motif as illustrated by electrostatic potential mapping (Fig. 1c).

Currently, the wHTH motif is described as a conserved structural element whose interaction profiles and functional properties in different proteins are extremely versatile, ranging from nucleic acid binding to protein-protein interactions ${ }^{6}$. Search for structural homologues using the DALI server ${ }^{7}$ and PDBeFold ${ }^{8}$ returned no matches for the entire structure but to other wHTHcontaining protein domains, whereby sharing a sequence identity of only $10-15 \%$, explaining the missed $\mathrm{wHTH}$ motif prediction. The DNA replication factor Cdt1 (PDB code 3a4c (ref. 9)), a key licensing factor that recruits the MCM2-7 complex onto the origin of chromosomes, is the closest structural homologue with a root-mean-square deviation (r.m.s.d.) of $3.2 \AA$ over $99 \alpha$-carbons (Supplementary Fig. 2a). Other structural similarity matches identified by the DALI ${ }^{7}$ search were wHTH domains found in E3 ubiquitin ligase complexes. The complex of the C-terminal domain of cullin-1 and the RING domain of the E3 ubiquitin ligase Rbx1 (ref. 10), for example, superimposes with an r.m.s.d. of $3.8 \AA$ over 79 residues onto the wHTH motif of the ROQ domain (Supplementary Fig. 2b). In the cullin-RING ligase complex, the wHTH subdomain of cullin-1 mediates contacts with the Rbx1 RING domain and similar interactions might be observed between the wHTH motif of the ROQ domain and the N-terminal RING domain of ROQUIN1. 
a

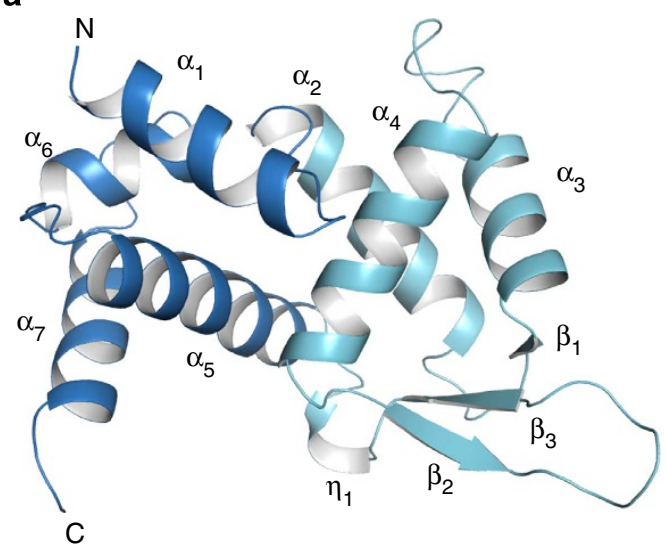

b

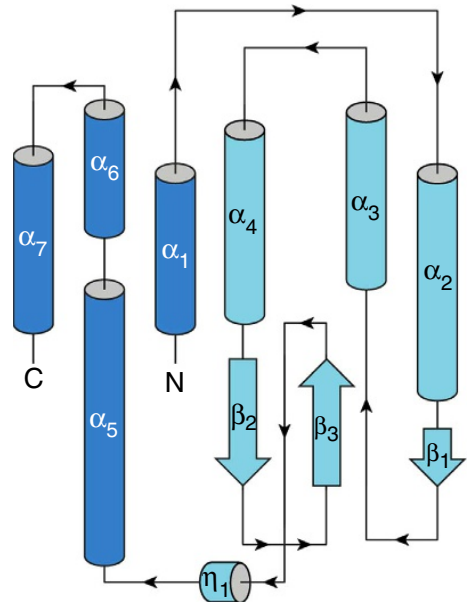

C

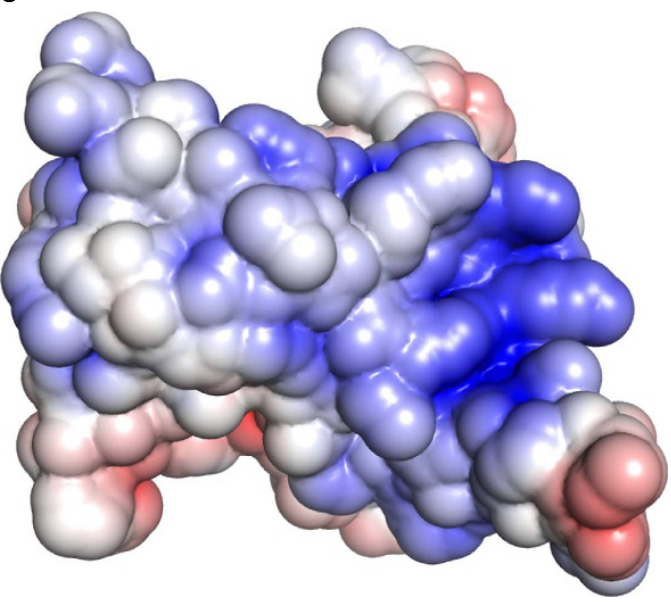

Figure 1 | Crystal structure of the ROQ domain of ROQUIN1. (a) Cartoon model of the overall fold with the wHTH motif highlighted in light blue. (b) Topological model of the ROQ domain fold. Colouring in panels $\mathbf{a}$ and $\mathbf{b}$ is identical. (c) The electrostatic surface of the ROQ domain (orientation as in a), calculated with the APBS plugin in Pymol ( $\pm 5 \mathrm{kT} / \mathrm{e}$; red, negative; blue, positive).

Notably, no matches to other proteins with classical wHTH motifs and reported RNA-binding properties were found among the top hits in the structural similarity search 7 . However, to date only few such proteins have been described, displaying diverse RNA recognition modes ${ }^{11-16}$. Among these, the selenocysteine tRNA-specific elongation factor SelB is the single example of a
wHTH-containing protein recognizing stem-loop mRNA. Its C-terminal domain encompasses four wHTH domains. The two terminal tandem wHTH motifs synergize to establish specific interactions with the selenocysteine incorporation site hairpin, and the last motif specifically recognizes the mRNA-specific hairpin tip structure of the mRNA ${ }^{12,16}$. The C-terminal SelB wHTH domain superimposes with an r.m.s.d. of $3.1 \AA$ over 73 residues onto the wHTH motif within the crystal structure of the ROQ domain, displaying a similar electropositive pocket as described for the ROQ domain crystal structure (Supplementary Fig. 2c).

ROQUIN1 binding to CDE-containing stem-loop mRNAs. Structural analysis indicated a role of the wHTH motif of the ROQ domain in RNA binding. To support this finding, we analysed the RNA-binding properties of different wHTH variants. Within the wHTH motif, single basic residues that may confer binding of the RNA backbone are highly conserved throughout the Roquin homologues. However, clusters of basic solventaccessible residues can be found at the N-terminal base of helix $\alpha 3$ (R219 and K220) and in the $\beta 2-\beta 3$ loop (K259 and R260) (Supplementary Fig. 3). To verify a potential involvement of these wHTH residues in mRNA binding, we performed electrophoretic mobility shift assays (EMSA) using wild-type and variant proteins comprising the RING, ROQ and $\mathrm{zf}$ domains of ROQUIN1 $\left(\mathrm{RC} 3 \mathrm{H1}^{2-452}\right)$, and the isolated $\mathrm{CDE}$-containing stem-loop structure of ICOS mRNA. Wild-type RC3H1 $1^{2-452}$ binds with a $K_{\mathrm{d}}$ value of $139 \pm 42 \mathrm{nM}$ to ICOS stem-loop mRNA (Fig. 2a), which is comparable with the binding constant reported for another CDE-containing stem-loop mRNA, namely TNF- $\alpha$ $(92 \pm 14 \mathrm{nM})^{1}$. On the other hand, $\mathrm{RC} 3 \mathrm{H}^{2-452}$ variants with single basic patch mutations (RK219AA or KR259AA, respectively) showed significantly reduced ICOS binding while simultaneous mutation of the two basic patches nearly abolished binding (Fig. 2a), clearly demonstrating an involvement of the wHTH motif in stem-loop mRNA binding. Notably, the observed effects are not due to altered secondary structures induced by the mutation as demonstrated by circular dichroism (CD) spectroscopy measurements (Supplementary Fig. 4).

Stem-loop RNA binding to dimeric ROQUIN1. ROQUIN1 purified as monomeric protein as judged by its gel filtration profile. To verify the oligomerization state in solution, we additionally performed sedimentation velocity ultracentrifugation experiments with the $\mathrm{RC} 3 \mathrm{H} 1^{2-452}$ protein, revealing the presence of $60 \%$ monomers, 33\% dimers and 7\% higher oligomers (Fig. 2b). This points towards the ability of ROQUIN1 to bind to RNA in various oligomerization states. To shed light on this, we next repeated the experiment in the presence of stem-loop ICOS RNA. All protein peaks are shifted to higher sedimentation coefficients on complex formation with RNA with no significant changes in the relative abundance of the various species, supporting the above hypothesis (Fig. 2b).

Analysis of the analytical ultracentrifugation experiments indicated that, in addition to monomeric protein, also ROQUIN1 dimers are able to bind to stem-loop RNA. Inspection of the ROQ domain crystal structure and adjacent symmetry-related segments by using ePISA ${ }^{17}$ revealed a potential dimer arrangement (Fig. 3a) that could allow for independent binding of two stem-loop RNA molecules to the two electropositive wHTH sites within the dimer (Fig. 3b), assuming that the stem-loop RNA-binding sites are identical in monomeric and dimeric ROQUIN1. Moreover, we consider this crystallographic dimer rather weak per se as indicated by the small buried surface area of $738 \AA^{2}$ with a limited number of hydrogen bonding and salt-bridge interactions at the dimer interface (Supplementary Table 1), and the fact that 
a

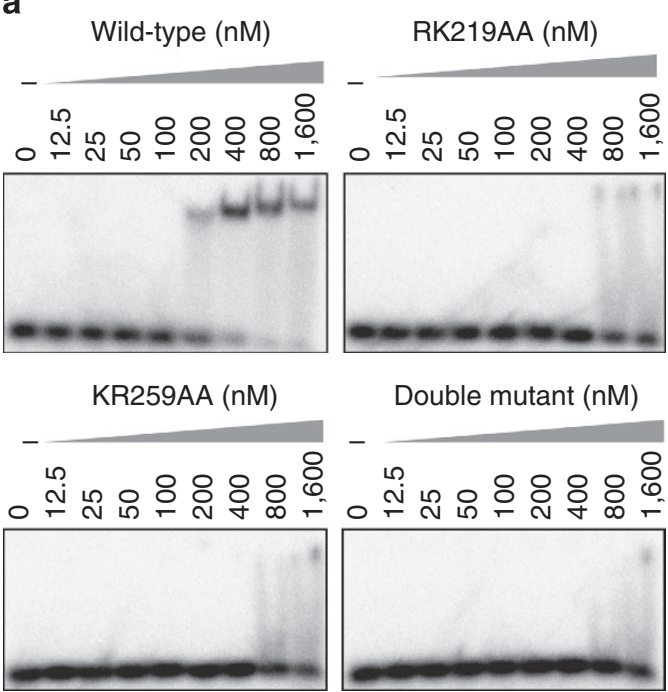

b

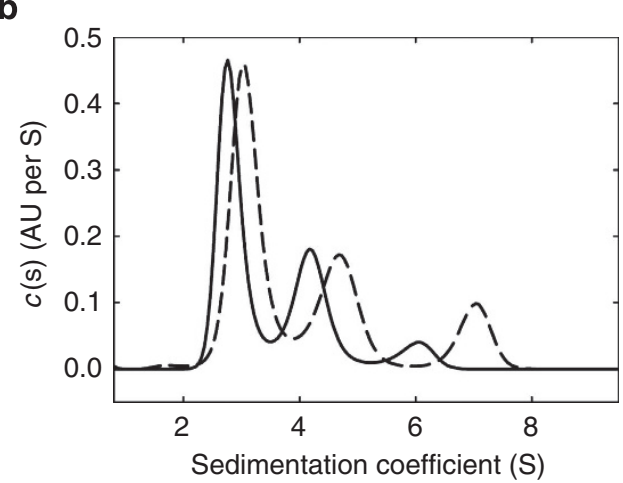

Figure 2 | ROQUIN1 stem-loop ICOS-mRNA binding studies. (a) EMSA experiments performed with the isolated CDE stem-loop structure of ICOS mRNA and wild type or variant $\mathrm{RC} 3 \mathrm{H}^{2-452}$. The double mutant corresponds to RK219AA/KR259AA of $\mathrm{RC} 3 \mathrm{H}^{2-452}$. The results are representative of at least two independent experiments. (b) Analytical ultracentrifugation analyses of uncomplexed $(-)$ and stem-loop ICOS RNA-complexed (---) $\mathrm{RC} 3 \mathrm{H} 1^{2-452}$ protein.

the dimeric fraction of uncomplexed ROQUIN1, whose existence has been indicated by our analytical ultracentrifugation experiments (Fig. 2b), is not stable under the separation shear forces on a gel filtration (Supplementary Fig. 5a).

To verify that this proposed dimer is capable of stem-loop RNA binding, we designed a dimer-stabilizing mutant and introduced two disulfide bridges at the dimer interface by generating the $\mathrm{P} 327 \mathrm{C} / \mathrm{T} 258 \mathrm{C}$ variant, which links the $\mathrm{C}$ terminus of the ROQ domain to the C-terminal end of $\beta 2$ within the wHTH motif. The double-cysteine variant purifies as a mixture of monomers and dimers, with the dimeric fraction being convertible to monomers on DTT treatment (Supplementary Fig. 5b) and with a secondary-structure content similar to wildtype protein (Supplementary Fig. 4). Strikingly, the disulfidestabilized dimer possesses a significantly increased stem-loop RNA affinity compared with wild-type protein as revealed by EMSA (Fig. 3c). Addition of DTT brings the RNA-binding ability back to wild-type level (Fig. 3c), supporting our proposed dimer model.

ROQUIN1 binding to duplex RNA. To further analyse the stoichiometry of RNA binding to ROQUIN1, we performed isothermal titration calorimetry (ITC) where we calculated an unexpected overall binding stoichiometry of around 0.5 , implying that two molecules of $\mathrm{RC} 3 \mathrm{H}_{1}{ }^{2-452}$ protein bind to one molecule of RNA (Fig. 3d). However, we realized that the concentrations applied during this ITC experiment involved RNA annealing at a rather high concentration yielding bulged duplex RNA instead of the stem-loop form (Supplementary Figs $5 \mathrm{c}$ and 6), a phenomenon that has also been described by Tan et al. ${ }^{18}$ Interestingly, we determined a $K_{\mathrm{d}}$ value of $68.9 \pm 8.6 \mathrm{nM}$ for the binding of $\mathrm{RC} 3 \mathrm{H}^{2-452}$ to ICOS duplex RNA (Fig. 3d), which is in the same range as observed for stem-loop RNA. Taken together, ROQUIN1 is not only able to bind to stem-loop but also to double-stranded RNA.

Results from analytical gel filtration experiments confirmed the 2:1 binding model indicated by the above ITC experiment. In the absence of duplex RNA, the calculated molecular mass corresponds to monomeric $\mathrm{RC}^{\mathrm{H}} \mathrm{H}^{2-452}$ protein, and the dimeric protein was found in the presence of duplex ICOS RNA (Supplementary Fig. 5a). Notably, we observe a complete shift of the monomeric fraction on duplex RNA addition despite adding only 0.6 -fold molar amount of duplex RNA to prepare the complex. The same holds true also for the crystallized $\mathrm{RC} 3 \mathrm{H} 1^{1 / 8-328}$ degradation fragment of ROQUIN1, indicating that the RING and zf domains of ROQUIN1 are dispensable for both RNA binding and dimerization (Supplementary Fig. 5d).

The latter finding hints towards the possibility that the proposed ROQ domain dimer arrangement (Fig. 3a) may also support duplex RNA binding. In accordance with the 2:1 binding stoichiometry and the electrostatic potential mapping (Fig. 3b), the putative duplex RNA-binding site is likely located at the dimer interface. Furthermore, the width of the binding groove at the dimer interface seems sufficient to accommodate doublestranded RNA. To prove our hypothesis, we performed an ITC experiment using the cysteine-bridged $\mathrm{P} 327 \mathrm{C} / \mathrm{T} 258 \mathrm{C}$ variant of $\mathrm{RC} 3 \mathrm{H1}^{2-452}$. Remarkably, a ROQUIN1 dimer stabilized this way binds to ICOS duplex RNA with the same 2:1 stoichiometry as described before (when referring to monomer protein in ITC calculations), substantiating the dimer model (Fig. 3d). Compared with wild type, the observed $\sim 10$-fold decreased $K_{\mathrm{d}}$ value for duplex ICOS binding to the stabilized ROQUIN1 dimer variant may be attributed to the decrease of flexibility in the rigidified cysteine-bridged dimer, which otherwise may be necessary for efficient duplex RNA binding at the dimer interface (Supplementary Fig. 7a).

Since the two basic patches within the wHTH motif contribute to the electropositive surface at the dimer interface, we next tested whether a variant with simultaneous mutation of these sites (RK219AA and KR259AA) would also interfere with duplex ICOS binding. Indeed, this variant nearly abolished duplex RNA binding (Supplementary Fig. 8), demonstrating that the wHTH motif within the ROQ domain is not only involved in stem-loop RNA binding but also has the potential to recognize doublestranded RNA.

Finally, we examined whether the ROQ domain dimerization is mediated predominantly by duplex RNA binding or by monomer-monomer contacts. ITC experiments show that the $\mathrm{RC} 3 \mathrm{H}^{2-452}$ variant proteins bearing mutations in the dimer interface bind double-stranded ICOS RNA with similar affinity as wild-type ROQUIN1 (Supplementary Fig. 8). This observation supports a model where ROQUIN1 dimers are formed following duplex RNA binding.

The capability of wHTH-containing proteins to bind to duplex RNAs at the interface of the two monomers has also been described for $Z \alpha$ domain-containing proteins that play a role in antiviral responses by recognizing foreign nucleic acids in the cytoplasm ${ }^{19}$. Its wHTH motif contacts five phosphates of a 
a

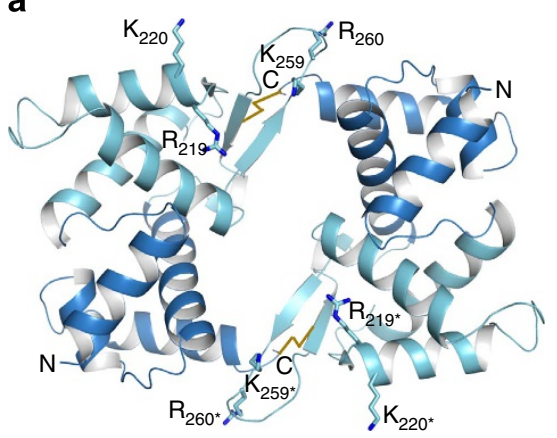

C

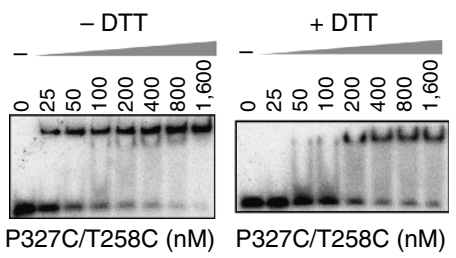

b

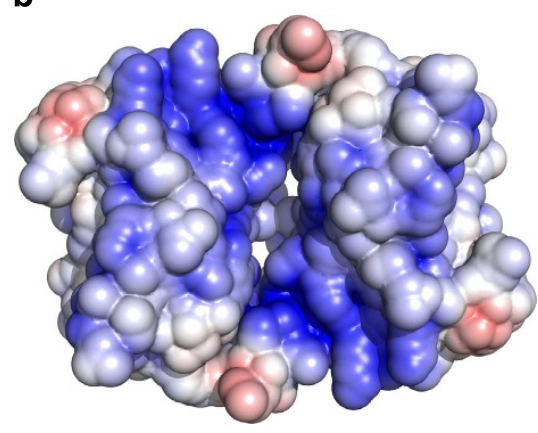

d

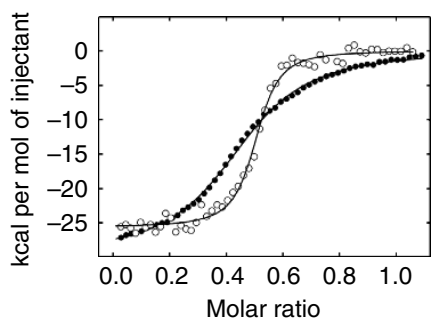

Figure 3 | Model of the dimeric ROQ domain, capable to bind to various RNA forms. (a) Cartoon model of the dimeric ROQ domain with the wHTH motif highlighted in light blue. Amino acids involved in RNA binding are labeled and shown in stick representation. Dimer-stabilizing disulfide bonds, generated by the P327C/T258C mutation, are shown in yellow. (b) The electrostatic surface of the dimeric ROQ domain (orientation as in panel a), calculated with the APBS plugin in Pymol ( $\pm 5 \mathrm{kT} / \mathrm{e}$; red, negative; blue, positive). (c) EMSA performed with the P327C/T258C variant of the RC3H12-452 protein and the isolated CDE stem-loop structure of ICOS mRNA. The results are representative of at least two independent experiments. (d) ITC isotherms characterizing the binding of wild type and P327C/T258C RC3H12-452 protein to duplex ICOS RNA. The data were fitted to a one-site binding model, revealing $K_{d}$ values of $68.9 \pm 8.6 \mathrm{nM}$ and $724.6 \pm 20.9 \mathrm{nM}$ and stoichiometry values of $n=0.499 \pm 0.003$ and $n=0.469 \pm 0.002$ for wild-type and cysteine-bridged dimer of $\mathrm{RC} 3 \mathrm{H}^{2-452}$, respectively, with errors referring to the s.e. of the fit.

left-handed duplex Z-RNA with residues from its $\mathrm{H} 3$ recognition helix and the W1-loop ${ }^{13}$. Moreover, two $\mathrm{Z} \alpha$ domains interact with the RNA duplex without forming a physical dimer, suggesting a cooperative mode of RNA-binding ${ }^{19}$.

\section{Discussion}

Our work provides insight into the mode of RNA binding by ROQUIN1. Combined structural and biochemical analyses substantiate the involvement of the wHTH motif of the ROQ domain in CDE-containing stem-loop mRNA binding. Furthermore, we illustrate that ROQUIN1 is additionally able to bind to doublestranded RNA and has the potential to be functional in various oligomerization states. Notably, the ROQ domain of ROQUIN1 appears to be sufficient both for RNA binding and dimerization. Finally, we propose a dimer model of the ROQ domain that accounts for stem-loop as well as duplex RNA binding.

During revision of this manuscript, two independent publications appeared describing structural aspects of roquin-RNA interactions ${ }^{18,20}$. The findings of these authors are in general agreement with the data presented here, particularly with regard to the involvement of the wHTH motif in CDE-containing stemloop RNA binding ${ }^{18,20}$, the ability of the ROQ domain to bind also RNA duplexes ${ }^{18}$ and to dimerize, either concentrationdependent ${ }^{20}$ or induced by duplex RNA binding ${ }^{18}$. The dimeric arrangement seen in the crystal structure of the ROQ domain bound to duplex RNA ${ }^{18}$ differs from our proposed dimer model. This difference may be attributed to the use of a different and longer RNA molecule, namely CDE-containing TNF23, in that study compared with the present work. To examine whether our dimer model is also relevant for $\mathrm{TNF}_{\mathrm{ds}}$ RNA binding, we performed EMSA experiments with the disulfide-stabilized $\mathrm{P} 327 \mathrm{C} / \mathrm{T} 258 \mathrm{C}$ dimer variant of $\mathrm{RC} 3 \mathrm{H} 1^{2-452}$ that again displays an increased affinity compared with wild-type protein (Supplementary Fig. 9a). Addition of DTT to disrupt disulfide links brings the dsRNA binding affinity back to wild-type level (Supplementary Fig. 9a). To rule out unspecific binding of the $\mathrm{TNF}_{\mathrm{ds}}$ RNA duplex, we finally tested the cysteine-bridged $\mathrm{RC} 3 \mathrm{H}_{1}{ }^{2-452}$ dimer for binding of the single-stranded RNA species of $\mathrm{TNF}_{\mathrm{ds}}$, which almost abolished binding, indicating the requirement of a duplex nature of the RNA for efficient binding by ROQUIN1 (Supplementary Fig. 9b). Conclusively, multiple modes of interaction with RNA might be possible, depending on the nature of the RNA (see also Supplementary Fig. 7b).

Whether the described dimeric states of Roquin proteins are physiologically relevant remains to be demonstrated. As indicated by our analytical ultracentrifugation experiments, monomeric ROQUIN1 predominates at lower protein concentrations, but a concentration-dependent dimerization of Roquin has been reported by Schlundt et al. ${ }^{20}$ Since Roquin proteins accumulate in $\mathrm{P}$ bodies and stress granules ${ }^{3,21}$, it is tempting to speculate that elevated Roquin concentration at these sites might favour the formation of functional dimers.

In conclusion, Roquin proteins turn out to be very versatile in terms of the secondary structure of their bound RNA targets. It is conceivable that in addition to the reported $\mathrm{CDE}$-containing stem-loop mRNAs also other structure-sequence motifs are recognized within targeted mRNAs, as also indicated by the fact that the majority of the mRNAs enriched in $\mathrm{RC} 3 \mathrm{H} 1$ immunoprecipitation experiments were lacking the CDE motif ${ }^{1}$. This opens up exciting prospects for future research, addressing further biological roles of Roquin family members.

\section{Methods}

Cloning, expression and purification. DNA encoding the RING, ROQ and $\mathrm{zf}$ domains of human ROQUIN1 (RC3H1 $1^{2-452}$, a $2-452$ ), the RING and ROQ 
domains ( $\mathrm{RC} 3 \mathrm{H} 1^{2-399}$, aa $\left.2-399\right)$, the ROQ domain ( $\mathrm{RC} 3 \mathrm{H} 1^{53-399}$, aa $\left.53-399\right)$ and the crystallized ROQ domain ( $\mathrm{RC} 3 \mathrm{H} 1^{178-328}$, aa $\left.178-328\right)$ was subcloned into the pQLinkH vector ${ }^{22}$. The following primers were used for cloning: RC3H1_2fw 5'-CAGGATCCCCTGTACAAGCTCCACAATGG-3', RC3H1_53fw 5'-GAG GAT CCG ACC AGA CCA CTA TCA ATA CAG ACA-3', RC3H1_178fw $5^{\prime}$-GAG GAT CCC AAC TCT CTT CCA ATC TTT GGG C- $3^{\prime}$, RC3H1_328rv $5^{\prime}$-GAC TGA CTG CGG CCG CTC AGG CTG GAG TCT GCA ACT TG-3', RC3H1_399rv 5'-GAC TGA CTG CGG CCG CTC AGC TGT GGT TCT GGA TAT AAT CAA CC- ${ }^{\prime}$, and RC3H1_452rv 5'-GAC TGA CTG CGG CCG CTC AGC GCT TAT TCA TTT TAC GAA ATT TTT CC- $3^{\prime}$. Mutants were generated using the QuikChange Site-Directed Mutagenesis Kit (Stratagene) according to the manufacturer's protocol. The genes were expressed as $\mathrm{N}$-terminal $\mathrm{His}_{7}$-tagged proteins at $17^{\circ} \mathrm{C}$ in E. coli Rosetta 2 (DE3) (Novagen) using a LEX ultra-high-throughput bench-top bioreactor (Harbinger Biotech). Cells were grown at $37^{\circ} \mathrm{C}$ in Terrific Broth medium and induced at an $\mathrm{OD}_{600}$ of $2.0-2.5$ with $0.5 \mathrm{mM}$ isopropyl $\beta$-D-1-thiogalactopyranoside. For purification, cells were resuspended in PBS lysis buffer $(1 \times \mathrm{PBS} \mathrm{pH}$ $7.4,0.5 \mathrm{M} \mathrm{NaCl}, 5 \%$ (v/v) glycerol, $0.5 \mathrm{mM}$ dithiothreitol (DTT)), supplemented with $0.25 \%(\mathrm{w} / \mathrm{v}) 3$-[(3-cholamidopropyl)dimethylammonio]-1-propanesulfonate, $0.1 \mathrm{mM}$ phenylmethyl sulfonyl fluoride, $1 \mathrm{U} \mathrm{ml}^{-1}$ RNase-free DNase I (Qiagen) and one tablet of EDTA-free Complete Protease Inhibitor (Roche). The purification protocol comprises mechanical cell lysis by sonication (SONOPULS HD 2200, Bandelin), Ni/Zn affinity chromatography on a $5 \mathrm{ml}$ HisTrap FF crude column (GE Healthcare) and size-exclusion chromatography on a Superdex 200 prep grade column (XK $26 \times 60$, GE Healthcare). The His $\mathrm{Hag}_{7}$ was cleaved with tobacco etch virus protease before the gel filtration step, followed by a reapplication of the cleaved protein on the Ni/Zn affinity column. The purification of protein constructs comprising the RING, ROQ and zf domains additionally included a cationexchange chromatography on a Source $30 \mathrm{~S}$ column (HR $16 \times 10$, GE Healthcare). The P327C/T258C variant was expressed in E. coli SHuffle express cells (NEB), and the buffers for initial purification steps included $3 \mathrm{mM}$ GSH (glutathione) and $0.6 \mathrm{mM}$ GSSG (glutathione disulfide).

Protein crystallization. The ROQ domain was crystallized using the sitting-drop vapour-diffusion method at $20^{\circ} \mathrm{C}$ by mixing equal volumes (200 nl) of $\mathrm{His}_{7}$ tag cleaved RC3H1 ${ }^{2-399}$ or RC3H1 ${ }^{53-399}$ protein $\left(8.5\right.$ or $10 \mathrm{mg} \mathrm{ml}^{-1}$ in $20 \mathrm{mM}$ HEPES/NaOH pH 7.5, $0.2 \mathrm{M} \mathrm{NaCl}, 5 \mathrm{mM}$ DTT, $\left.\pm 1 \mathrm{mM}\left(\mathrm{NH}_{4}\right)_{2} \mathrm{PtCl}_{4}\right)$ and reservoir solution (Pt derivative: $16 \% \mathrm{w} / \mathrm{v}$ PEG $6000,10 \mathrm{mM}$ sodium citrate; native: $20 \%$ (w/v) PEG 6000, $0.2 \mathrm{M}$ lithium chloride, $0.1 \mathrm{M}$ Tris/HCl pH 8.0). Before flashfreezing in liquid nitrogen, the crystal was transferred into a cryoprotectant consisting of reservoir solution supplemented with $30 \%(\mathrm{v} / \mathrm{v})$ glycerol. For derivatization, $\left.0.2 \mu \mathrm{l} 0.5 \mathrm{M}\left(\mathrm{NH}_{4}\right)_{2} \mathrm{PtCl}_{4}\right)$ was added to the crystallization drop before transfer to the cryoprotectant.

Data collection, structure determination and refinement. Diffraction data were collected on BL14.1 operated by the Helmholtz-Zentrum Berlin (HZB) at the BESSY II electron storage ring (Berlin-Adlershof, Germany) ${ }^{23}$ at wavelengths of 1.0727 or $0.9184 \AA$, respectively. Data were processed with the program XDSAPP ${ }^{24}$. Platinum derivative crystals were used for phasing by single-wavelength anomalous diffraction. Two peak data sets collected from the same crystal were merged with $\mathrm{XSCALE}^{25}$ to increase multiplicity. The platinum substructure was solved using $\mathrm{HKL}_{2} \mathrm{MAP}^{26}$, and the phases derived from the identified platinum positions were used for density modification as implemented in the PHENIx AutoSol wizard ${ }^{27}$. Autobuilding of an initial model and refinement were done with PHENIX ${ }^{28}$. The graphics program $\operatorname{coot}^{29}$ was used for model building and visualization. The resulting model was transferred to the high-resolution native data by molecular replacement using the program PHASER ${ }^{27}$ and refined with REFMAC ${ }^{30}$. Data collection and refinement statistics are reported in Table 1. The Ramachandran map produced by MolProbity ${ }^{31}$ shows that $98.7 \%$ of the modelled residues are in favoured regions with no outliers.

Structure evaluation. Secondary-structure elements were assigned using the program DSSP ${ }^{32}$. Searches for similar structures were performed with PDBeFold ${ }^{8}$ and the DALI-Server ${ }^{7}$. Interfaces present in the crystal were analysed using the web service ePISA ${ }^{17}$. Molecular drawings were created with PyMOL Molecular Graphics System (Version 1.3, Schrödinger, LLC). APBS was used for calculating electrostatic surface potentials ${ }^{33}$.

Multiple sequence alignment. Amino-acid sequences were aligned using the Clustal Omega server ${ }^{34}$, and TEXshade ${ }^{35}$ was used for illustration. Uniprot accession numbers are as follows. RC3H1: Homo sapiens, Q5TC82; Mus musculus, Q4VGL6; Rattus norvegicus, D3ZKG8; Gallus gallus, E1BZI9; Xenopus laevis, Q6NUC6. RC3H2: Homo sapiens, Q9HBD1; Mus musculus, P0C090; Rattus norvegicus, D3ZBM2; Gallus gallus, F1NZ02. Roq: Drosophila melanogaster, Q9VV48.

Isothermal titration calorimetry. ITC measurements were performed at $15^{\circ} \mathrm{C}$ in $20 \mathrm{mM}$ Tris/HCl, $\mathrm{pH} 8.0$ and $60 \mathrm{mM} \mathrm{KCl}$ using a VP-ITC microcalorimeter (MicroCal Inc.). Before the measurement, protein and synthetic RNA (ICOS_13nt:
5'-AUUUC UGUGA AAU-3' (Biomers); annealed at about $750 \mu \mathrm{M}$ ) were dialysed against this buffer. Experiments were performed by injecting $55 \times 5 \mu$ of $75-$ $100 \mu \mathrm{M}$ duplex ICOS RNA solution into a sample cell containing $15-20 \mu \mathrm{M}$ wildtype or variant of $\mathrm{RC} 3 \mathrm{H}^{2-452}$. Injections were performed with a spacing of $240 \mathrm{~s}$ and a reference power of $18 \mu \mathrm{cal} \mathrm{s}^{-1}$. Integration of the injection peaks and construction of binding isotherms was performed by using the high-precision automated peak shape analysis software NITPIC 1.0.3 (ref. 36). Data analysis was performed with the Origin 7.0 software (MicroCal, USA) using a one-site binding model.

Analytical gel filtration. Absolute molecular masses of the proteins and RNA complexes were determined by analytical gel filtration using a calibrated 10/300 Superdex 200 column. The running buffer contained $20 \mathrm{mM}$ HEPES/NaOH, $\mathrm{pH}$ 7.5 and $0.1 \mathrm{M} \mathrm{NaCl}$. For each protein sample, $50-100 \mu \mathrm{l}$ of a $1-5 \mathrm{mg} \mathrm{ml}^{-1}$ protein solution was applied. RNA complexes were prepared by adding the 0.6 -fold amount of duplex ICOS_13nt RNA (annealed at about $750 \mu \mathrm{M}$ ) to the protein. The P327C/T258C mutant of RC3H1 $1^{2-452}$ was analysed with/without $30 \mathrm{~min}$ preincubation with $100 \mathrm{mM}$ DTT at room temperature.

Circular dichroism spectroscopy. Wild-type or variant $\mathrm{RC} 3 \mathrm{H} 1^{2-452}$ proteins were dialysed against $10 \mathrm{mM}$ sodium phosphate buffer $\mathrm{pH} 7.5$ and $20 \mathrm{mM} \mathrm{NaF}$ and diluted to $2 \mu \mathrm{M}$. Spectra were recorded in three replicates at $20^{\circ} \mathrm{C}$ from $260 \mathrm{~nm}$ to $185 \mathrm{~nm}$, with a $0.1 \mathrm{~nm}$ step size using a Chirascan CD spectrophotometer (Applied Photophysics).

Electrophoretic mobility shift assay. The EMSA experiments were performed according to Ryder et al. ${ }^{37}$ with the following modifications: RNA was prepared by $5^{\prime}$ end-labelling of commercially synthesized RNA oligonucleotides with $\left[\gamma^{-32} \mathrm{P}\right]-$ ATP using T4 polynucleotide kinase (NEB). Labeled RNA was gel-purified, eluted and adjusted with water to $0.5 \mathrm{pmol}_{\mathrm{Hl}^{-1}}$. $350 \mathrm{fmol}$ of labeled RNA was used per $20 \mu \mathrm{l}$ reaction. Before binding reactions, a master mix containing labeled RNA, $1 \times$ binding buffer ( $20 \mathrm{mM}$ Tris- $\mathrm{HCl} \mathrm{pH} \mathrm{7.5,50} \mathrm{mM} \mathrm{KCl,} 5 \mathrm{mM} \mathrm{MgCl}_{2}, 20 \mu \mathrm{M} \mathrm{ZnSO}_{4}$ $10 \%$ glycerol), $2 \mathrm{mM} \mathrm{DTT}$ and $0.05 \mathrm{mg} \mathrm{ml}^{-1} \mathrm{BSA}$ was heated at $90^{\circ} \mathrm{C}$ for $1 \mathrm{~min}$ and gradually cooled down to room temperature. In parallel, a dilution series of $10 \times$ protein stock was prepared in $1 \times$ protein dilution buffer $(1 \times$ binding buffer, $5 \mu \mathrm{g} \mathrm{ml}^{-1}$ heparin). For each binding reaction, $2 \mu \mathrm{l}$ of the $10 \times$ protein stock was added to $18 \mu \mathrm{l}$ of the master mix at room temperature for $2-3 \mathrm{~h}$. After addition of $4 \mu \mathrm{l} 6 \times$ loading buffer ( $30 \%$ glycerol, bromphenol blue, xylene cyanol), RNP complexes were resolved by non-denaturing PAGE ( $6 \%$ polyacrylamide, $0.5 \times$ TBE, $5 \%$ glycerol) in ice-cold $0.5 \times$ TBE buffer containing $20 \mu \mathrm{MnSO}_{4}$ at $100 \mathrm{~V}$ for $40 \mathrm{~min}$. The protein-bound RNA and the free RNA were imaged using a phosphorimager. The P327C/T258C mutant of RC3H1 $1^{2-452}$ was analysed with/ without $30 \mathrm{~min}$ preincubation with $10 \mathrm{mM}$ DTT at room temperature. The results are representative of two to four independent experiments. For $K_{\mathrm{d}}$ calculation, the average of bound fraction from three independent experiments was normalized to the total signal for each lane and plotted against the protein concentration. The data points were fitted by nonlinear regression with errors referring to the s.e. of the fit.

Analytical ultracentrifugation. Sedimentation velocity experiments were carried out at 35,000 r.p.m. in an XLI analytical ultra-centrifuge (Beckman). For all samples, $400 \mu \mathrm{l}$ of RC3 $\mathrm{Hl}^{2-452}$ protein at a concentration of $0.3 \mathrm{mg} \mathrm{ml}^{-1} \pm 1.1$-fold excess of stem-loop ICOS RNA (annealed at $2 \mu \mathrm{M}$ ) and reference buffer $(20 \mathrm{mM}$ Hepes pH 7.5, $0.1 \mathrm{M} \mathrm{NaCl}$ ) were loaded in two-channel center pieces with an optical path length of $12 \mathrm{~mm}$. Samples were measured at $15^{\circ} \mathrm{C}$ in an An-50 Ti rotor using the interference optical system. The program SEDNTERP was used to estimate the partial specific volume from amino-acid composition as well as the density $\rho$ and viscosity $\eta$ of the buffer at $15^{\circ} \mathrm{C}$ (http:// bitcwiki.sr.unh.edu/index.php/ Main_Page). Data were then analysed with the program SEDFIT ${ }^{38}$ using a continuous $c(s)$ distribution model. Theoretical sedimentation coefficients for monomeric and higher oligomeric protein species were calculated using the following equation

$$
s=\frac{M_{\mathrm{W}}(1-\rho)}{N_{\mathrm{A}}\left(f / f_{0}\right) 6 \pi \eta \sqrt[3]{3 M / \pi N_{\mathrm{A}}}}
$$

with $N_{\mathrm{A}}$ Avogadro's number and $M_{\mathrm{W}}$ and $f / f_{0}$ the protein's molecular weight and frictional ratio, respectively. Assuming a frictional ratio of 1.5 , sedimentation coefficients of $2.8,4.4$ and 5.8 or $2.9,4.6$, and 6.1 were calculated for monomeric, dimeric or trimeric uncomplexed protein or protein-RNA complexes, respectively. The monomeric uncomplexed protein has a molecular weight of $50.9 \mathrm{kDa}$ for monomers, the monomeric protein-RNA complex is about $55 \mathrm{kDa}$.

\section{References}

1. Leppek, K. et al. Roquin promotes constitutive mRNA decay via a conserved class of stem-loop recognition motifs. Cell 153, 869-881 (2013).

2. Yu, D. et al. Roquin represses autoimmunity by limiting inducible T-cell costimulator messenger RNA. Nature 450, 299-303 (2007). 
3. Glasmacher, E. et al. Roquin binds inducible costimulator mRNA and effectors of mRNA decay to induce microRNA-independent post-transcriptional repression. Nat. Immunol. 11, 725-733 (2010).

4. Vogel, K. U. et al. Roquin paralogs 1 and 2 redundantly repress the Icos and Ox40 costimulator mRNAs and control follicular helper $\mathrm{T}$ cell differentiation. Immunity 38, 655-668 (2013).

5. Pratama, A. et al. Roquin-2 shares functions with its paralog Roquin-1 in the repression of mRNAs controlling $\mathrm{T}$ follicular helper cells and systemic inflammation. Immunity 38, 669-680 (2013).

6. Harami, G. M., Gyimesi, M. \& Kovács, M. From keys to bulldozers: expanding roles for winged helix domains in nucleic-acid-binding proteins. Trends Biochem. Sci. 38, 364-371 (2013).

7. Holm, L. \& Rosenström, P. Dali server: conservation mapping in 3D. Nucleic Acids Res. 38, W545-W549 (2010).

8. Krissinel, E. \& Henrick, K. Secondary-structure matching (SSM), a new tool for fast protein structure alignment in three dimensions. Acta Crystallogr. D Biol. Crystallogr. 60, 2256-2268 (2004).

9. Khayrutdinov, B. I. et al. Structure of the Cdt1 C-terminal domain: conservation of the winged helix fold in replication licensing factors. Protein Sci. 18, 2252-2264 (2009).

10. Calabrese, M. F. et al. A RING E3-substrate complex poised for ubiquitin-like protein transfer: structural insights into cullin-RING ligases. Nat. Struct. Mol. Biol. 18, 947-949 (2011).

11. Alfano, C. et al. Structural analysis of cooperative RNA binding by the La motif and central RRM domain of human La protein. Nat. Struct. Mol. Biol. 11, 323-329 (2004).

12. Yoshizawa, S. et al. Structural basis for mRNA recognition by elongation factor SelB. Nat. Struct. Mol. Biol. 12, 198-203 (2005).

13. Placido, D., Brown, B. A., Lowenhaupt, K., Rich, A. \& Athanasiadis, A. A lefthanded RNA double helix bound by the $\mathrm{Z}$ alpha domain of the RNA-editing enzyme ADAR1. Structure 15, 395-404 (2007).

14. Matsumoto, Y. et al. Structure of a virulence regulatory factor CvfB reveals a novel winged helix RNA binding module. Structure 18, 537-547 (2010).

15. Anantharaman, V., Zhang, D. \& Aravind, L. OST-HTH: a novel predicted RNA-binding domain. Biol. Direct 5, 13 (2010).

16. Soler, N., Fourmy, D. \& Yoshizawa, S. Structural insight into a molecular switch in tandem winged-helix motifs from elongation factor SelB. J. Mol. Biol. 370, 728-741 (2007).

17. Krissinel, E. \& Henrick, K. Inference of macromolecular assemblies from crystalline state. J. Mol. Biol. 372, 774-797 (2007).

18. Tan, D., Zhou, M., Kiledjian, M. \& Tong, L. The ROQ domain of Roquin recognizes mRNA constitutive-decay element and double-stranded RNA. Nat. Struct. Mol. Biol. 21, 679-685 (2014).

19. Athanasiadis, A. Zalpha-domains: at the intersection between RNA editing and innate immunity. Semin. Cell Dev. Biol. 23, 275-280 (2012).

20. Schlundt, A. et al. Structural basis for RNA recognition in roquin-mediated post-transcriptional gene regulation. Nat. Struct. Mol. Biol. 21, 671-678 (2014).

21. Athanasopoulos, V. et al. The ROQUIN family of proteins localizes to stress granules via the ROQ domain and binds target mRNAs. FEBS J. 277, 2109-2127 (2010).

22. Scheich, C., Kümmel, D., Soumailakakis, D., Heinemann, U. \& Büssow, K. Vectors for co-expression of an unrestricted number of proteins. Nucleic Acids Res. 35, e43-e43 (2007).

23. Mueller, U. et al. Facilities for macromolecular crystallography at the Helmholtz-Zentrum Berlin. J. Synchrotron Radiat. 19, 442-449 (2012)

24. Krug, M., Weiss, M. S., Heinemann, U. \& Mueller, U. XDSAPP: a graphical user interface for the convenient processing of diffraction data using XDS. J. Appl. Cryst. 45, 568-572 (2012).

25. Kabsch, W. XDS. Acta Crystallogr. D Biol. Crystallogr. 66, 125-132 (2010).

26. Pape, T. \& Schneider, T. R. HKL2MAP: a graphical user interface for phasing with SHELX programs. J. Appl. Cryst. 37, 843-844 (2004).

27. McCoy, A. J., Grosse-Kunstleve, R. W., Storoni, L. C. \& Read, R. J. Likelihoodenhanced fast translation functions. Acta Crystallogr. D Biol. Crystallogr. 61, 458-464 (2005)
28. Adams, P. D. et al. PHENIX: a comprehensive Python-based system for macromolecular structure solution. Acta Crystallogr. D Biol. Crystallogr. 66, 213-221 (2010).

29. Emsley, P., Lohkamp, B., Scott, W. G. \& Cowtan, K. Features and development of Coot. Acta Crystallogr. D Biol. Crystallogr. 66, 486-501 (2010).

30. Murshudov, G. N., Vagin, A. A. \& Dodson, E. J. Refinement of macromolecular structures by the maximum-likelihood method. Acta Crystallogr. D Biol. Crystallogr. 53, 240-255 (1997).

31. Davis, I. W. et al. MolProbity: all-atom contacts and structure validation for proteins and nucleic acids. Nucleic Acids Res. 35, W375-W383 (2007).

32. Kabsch, W. \& Sander, C. Dictionary of protein secondary structure: pattern recognition of hydrogen-bonded and geometrical features. Biopolymers 22, 2577-2637 (1983).

33. Baker, N. A., Sept, D., Joseph, S., Holst, M. J. \& McCammon, J. A. Electrostatics of nanosystems: application to microtubules and the ribosome. Proc. Natl Acad. Sci. USA 98, 10037-10041 (2001).

34. Sievers, F. et al. Fast, scalable generation of high-quality protein multiple sequence alignments using Clustal Omega. Mol. Syst. Biol. 7, 539 (2011).

35. Beitz, E. TEXshade: shading and labeling of multiple sequence alignments using LATEX2 epsilon. Bioinformatics 16, 135-139 (2000).

36. Keller, S. et al. High-precision isothermal titration calorimetry with automated peak-shape analysis. Anal. Chem. 84, 5066-5073 (2012).

37. Ryder, S. P., Recht, M. I. \& Williamson, J. R. Quantitative analysis of protein-RNA interactions by gel mobility shift. Methods Mol. Biol. 488, 99-115 (2008).

38. Schuck, P. Size-distribution analysis of macromolecules by sedimentation velocity ultracentrifugation and lamm equation modeling. Biophys. J. 78, 1606-1619 (2000).

\section{Acknowledgements}

The Protein Sample Production Facility at the Max Delbrück Center is funded by the Helmholtz Association of German Research Centres. The research group of M.L. is funded by the Federal Ministry for Education and Research (BMBF) and the Senate of Berlin, Berlin, Germany. Y.M. is funded by the Deutscher Akademischer Austauschdienst (DAAD). We thank Tracy Dornblut, Janett Tischer and Mandy Mustroph for excellent technical assistance, Kathrin Schulte for supporting the analytical gel filtration measurements, and Michael Krug and Yvette Roske for assistance with diffraction data collection. We acknowledge the beamline support by the staff of the Helmholtz-Zentrum Berlin für Materialien und Energie at BESSY.

\section{Author contributions}

A.S. and U.H. designed the study and guided the research experiments. A.S. carried out all the experiments except for EMSA and analytical ultracentrifugation experiments that were performed by Y.M. and E.R., respectively. All authors analysed the data. A.S. wrote and U.H., M.L. and Y.M. revised the manuscript.

\section{Additional information}

Accession codes: Coordinates and structure factors for the ROQ domain of human RC3H1/ROQUIN1 have been deposited in the Protein Data Bank under accession code 4ULW.

Supplementary Information accompanies this paper at http://www.nature.com/ naturecommunications

Competing financial interests: The authors declare no competing financial interests.

Reprints and permission information is available online at http://npg.nature.com/ reprintsandpermissions/

How to cite this article: Schuetz, A. et al. Roquin binding to target mRNAs involves a winged helix-turn-helix motif. Nat. Commun. 5:5701 doi: 10.1038/ncomms6701 (2014). 